This ECCV 2018 paper, provided here by the Computer Vision Foundation, is the author-created version.

The content of this paper is identical to the content of the officially published ECCV 2018

LNCS version of the paper as available on SpringerLink: https://ink.springer.com/conference/eccv

\title{
Recognition in Terra Incognita
}

\author{
Sara Beery, Grant Van Horn, and Pietro Perona \\ Caltech \\ \{sbeery, gvanhorn, perona\}@caltech.edu
}

\begin{abstract}
It is desirable for detection and classification algorithms to generalize to unfamiliar environments, but suitable benchmarks for quantitatively studying this phenomenon are not yet available. We present a dataset designed to measure recognition generalization to novel environments. The images in our dataset are harvested from twenty camera traps deployed to monitor animal populations. Camera traps are fixed at one location, hence the background changes little across images; capture is triggered automatically, hence there is no human bias. The challenge is learning recognition in a handful of locations, and generalizing animal detection and classification to new locations where no training data is available. In our experiments state-of-the-art algorithms show excellent performance when tested at the same location where they were trained. However, we find that generalization to new locations is poor, especially for classification systems. ${ }^{1}$
\end{abstract}

Keywords: Recognition, transfer learning, domain adaptation, context, dataset, benchmark.

\section{Introduction}

Automated visual recognition algorithms have recently achieved human expert performance at visual classification tasks in field biology $[1-3]$ and medicine $[4,5]$. Thanks to the combination of deep learning $[6,7]$, Moore's law [8] and very large annotated datasets $[9,10]$ enormous progress has been made during the past 10 years. Indeed, 2017 may come to be remembered as the year when automated visual categorization surpassed human performance.

However, it is known that current learning algorithms are dramatically less data-efficient than humans [11], transfer learning is difficult [12], and, anecdotally, vision algorithms do not generalize well across datasets [13,14] (Fig. 1). These observations suggest that current algorithms rely mostly on rote patternmatching, rather than abstracting from the training set 'visual concepts' [15] that can generalize well to novel situations. In order to make progress we need datasets that support a careful analysis of generalization, dissecting the challenges in detection and classification: variation in lighting, viewpoint, shape, photographer's choice and style, context/background. Here we focus on the latter: generalization to new environments, which includes background and overall lighting conditions.

1 The dataset is available at https://beerys.github.io/CaltechCameraTraps/ 


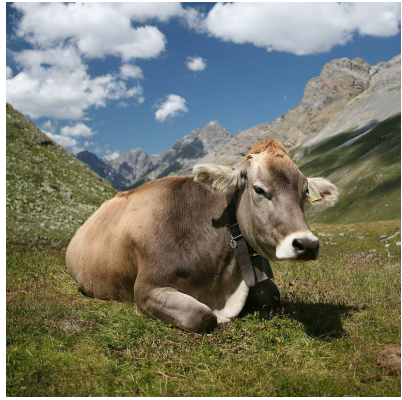

(A) Cow: 0.99, Pasture: 0.99, Grass: 0.99, No Person: 0.98, Mammal: 0.98

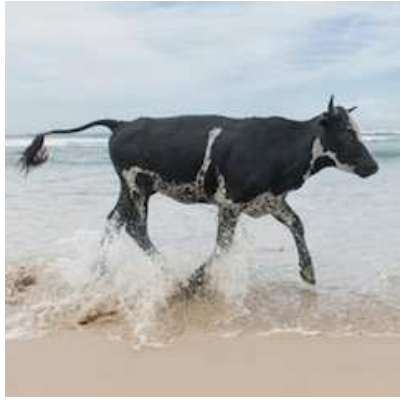

(B) No Person: 0.99, Water:

0.98, Beach: 0.97, Outdoors: 0.97 , Seashore: 0.97

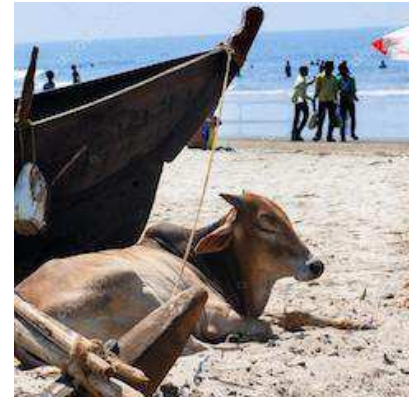

(C) No Person: 0.97,

Mammal: 0.96, Water: 0.94, Beach: 0.94, Two: 0.94

Fig. 1. Recognition algorithms generalize poorly to new environments. Cows in 'common' contexts (e.g. Alpine pastures) are detected and classified correctly (A), while cows in uncommon contexts (beach, waves and boat) are not detected (B) or classified poorly (C). Top five labels and confidence produced by ClarifAI.com shown.

Applications where the ability to generalize visual recognition to new environments is crucial include surveillance, security, environmental monitoring, assisted living, home automation, automated exploration (e.g. sending rovers to other planets). Environmental monitoring by means of camera traps is a paradigmatic application. Camera traps are heat- or motion-activated cameras placed in the wild to monitor and investigate animal populations and behavior. Camera traps have become inexpensive, hence hundreds of them are often deployed for a given study, generating a deluge of images. Automated detection and classification of animals in images is a necessity. The challenge is training animal detectors and classifiers from data coming from a few pilot locations such that these detectors and classifiers will generalize to new locations. Camera trap data is controlled for environment including lighting (the cameras are static, and lighting changes systematically according to time and weather conditions), and eliminates photographer bias (the cameras are activated automatically).

Camera traps are not new to the computer vision community [16-27,2]. Our work is the first to identify camera traps as a unique opportunity to study generalization, and we offer the first study of generalization to new environments in this controlled setting. We make here three contributions: (a) a novel, wellannotated dataset to study visual generalization across locations, (b) a benchmark to measure algorithms' performance, and (c) baseline experiments establishing the state of the art. Our aim is to complement current datasets utilized by the vision community for detection and classification $[9,10,28,29]$ by introducing a new dataset and experimental protocol that can be used to systematically evaluate the generalization behavior of algorithms to novel environments. In this work we benchmark the current state-of-the-art detection and classification pipelines and find that there is much room for improvement. 


\section{Related Work}

\subsection{Datasets}

The ImageNet [9], MS-COCO [10], PascalVOC [28], and Open Images [29] datasets are commonly used for benchmarking classification and detection algorithms. Images in these datasets were collected in different locations by different people, which enables algorithms to average over photographer style and irrelevant background clutter. However, as demonstrated in Fig. 1, the context can be strongly biased. Human photographers are biased towards well-lit, wellfocused images where the subjects are centered in the frame [30,31]. Furthermore, the number of images per class is balanced, unlike what happens in the real world [11].

Natural world datasets such as the iNaturalist dataset [1], CUB200 [32], Oxford Flowers [33], LeafSnap [34], and NABirds700 [35] are focused on finegrained species classification and detection. Most images in these datasets are taken by humans under relatively good lighting conditions, though iNaturalist does contain human-selected camera trap images. Many of these datasets exhibit real-world long-tailed distributions, but in all cases there is a large amount of diversity in location and perspective.

The Snapshot Serengeti dataset [21] is a large, multi-year camera trap dataset collected at 225 locations in a small region of the African savanna. It is the single largest-scale camera trap dataset ever collected, with over 3 million images. However, it is not yet suitable for controlled experiments. This dataset was collected from camera traps that fire in sequences of 3 for each motion trigger, and provides species annotation for groups of images based on a time threshold. This means that sometimes a single species annotation is provided for up to 10 frames, when in fact the animal was present in only a few of those frames (no bounding boxes are provided). Not all camera trap projects are structured in a similar way, and many cameras take shorter sequences or even single images on each trigger. In order to find a solution that works for new locations regardless of the camera trap parameters, it is important to have information about which images in the batch do or do not contain animals. In our dataset we provide annotations on a per-instance basis, with bounding boxes and associated classes for each animal in the frame.

\subsection{Detection}

Since camera traps are static, detecting animals in the images could be considered either a change detection or foreground detection problem. Detecting changes and/or foreground vs. background in video is a well studied problem [36], [37]. Many of these methods rely on constructing a good background model that updates regularly, and thus degrade rapidly at low frame rates. [38] and [39] consider low frame rate change detection in aerial images, but in these cases there are often very few examples per location. 
Some camera traps collect a short video when triggered instead of a sequence of frames. $[20,23,22]$ show foreground detection results on camera trap video. Data that comes from most camera traps take sequences of frames at each trigger at a frame rate of $\sim 1$ frame per second. This data can be considered "video," albeit with extremely low, variable frame rate. Statistical methods for background subtraction and foreground segmentation in camera trap image sequences have been previously considered. [16] demonstrates a graph-cut method that uses background modeling and foreground object saliency to segment foreground in camera trap sequences. [24] creates background models and perform a superpixel-based comparison to determine areas of motion. [25] uses a multilayer RPCA-based method applied to day and night sequences. [26] uses several statistical background-modeling approaches as additional signal to improve and speed up deep detection. These methods rely on a sequence of frames at each trigger to create appropriate background models, which are not always available. None of these methods demonstrate results on locations outside of their training set.

\subsection{Classification}

A few studies tackle classification of camera trap images. [18] showed results classifying squirrels vs. tortoises in the Mojave Desert. [17] showed classification results on data that provides image sequences of $\tilde{1} 0$ frames. They do not consider the detection problem and instead manually crop the animal from the frame and balance the dataset, resulting in a total of 7,196 images across 18 species with at least 100 examples each. [19] were the first to take a deep network approach to camera trap classification, working with data from eMammal [40]. They first performed detection using the background subtraction method described in [16], then classified cropped detected regions, getting $38.31 \%$ top- 1 accuracy on 20 common species. [27] show classification results on both Snapshot Serengeti and data from jungles in Panama, and saw a boost in classification performance from providing animal segmentations. [2] show 94.9\% top-1 accuracy using an ensemble of models for classification on the Snapshot Serengeti dataset. None of the previous works show results on unseen test locations.

\subsection{Generalization and Domain Adaptation}

Generalizing to a new location is an instance of domain adaptation, where each location represents a domain with its own statistical properties such as types of flora and fauna, species frequency, man-made or other clutter, weather, camera type, and camera orientation. There have been many methods proposed for domain adaptation in classification [41]. [42] proposed a method for unsupervised domain adaptation by maximizing domain classification loss while minimizing loss for classifying the target classes. We generalized this method to multi-domain for our dataset, but did not see any improvement over the baseline. [43] demonstrated results of a similar method for fine-grained classification, 
using a multi-task setting where the adaptation was from clean web images to real-world images, and [44] investigated open-set domain adaptation.

Few methods have been proposed for domain adaptation outside of classification. [45-47] investigate methods of domain adaptation for semantic segmentation, focusing mainly on cars and pedestrians and either adapting from synthetic to real data, from urban to suburban scenes, or from PASCAL to a camera onboard a car. [48-52] look at methods for adapting detectors from one data source to another, such as from synthetic to real data or from images to video. Raj, et. al., [53] demonstrated a subspace-based detection method for domain adaptation from PASCAL to COCO.

\section{The Caltech Camera Traps Dataset}

The Caltech Camera Traps (CCT) dataset contains 243,187 images from 140 camera locations, curated from data provided by the USGS and NPS. Our goal in this paper is to specifically target the problem of generalization in detection and classification. To this end, we have randomly selected 20 camera locations from the American Southwest to study in detail. By limiting the geographic region, the flora and fauna seen across the locations remain consistent. The current task is not to deal with entirely new regions or species, but instead to be able to recognize the same species of animals in the same region with a different camera background. In the future we plan to extend this work to recognizing the same species in new regions, and to the open-set problem of recognizing never-before-seen species. Examples of data from different locations can be seen in Fig. 2.

Camera traps are motion- or heat-triggered cameras that are placed in locations of interest by biologists in order to monitor and study animal populations and behavior. When a camera is triggered, a sequence of images is taken at approximately one frame per second. Our dataset contains sequences of length $1-5$. The cameras are prone to false triggers caused by wind or heat rising from the ground, leading to empty frames. Empty frames can also occur if an animal moves out of the field of view of the camera while the sequence is firing. Once a month, biologists return to the cameras to replace the batteries and change out the memory card. After it has been collected, experts manually sort camera trap data to categorize species and remove empty frames. The time required to sort and label images by hand severely limits data scale and research productivity. We have acquired and further curated a portion of this data to analyze generalization behaviors of state-of-the-art classifiers and detectors.

The dataset in this paper, which we call Caltech Camera Traps-20 (CCT-20), consists of 57,868 images across 20 locations, each labeled with one of 15 classes (or marked as empty). Classes are either single species (e.g. "Coyote" or groups of species, e.g. "Bird"). See Fig. 4 for the distribution of classes and images across locations. We do not filter the stream of images collected by the traps, rather this is the same data that a human biologist currently sifts through. Therefore 
the data is unbalanced in the number of images per location, distribution of species per location, and distribution of species overall (see Fig. 4).

\subsection{Detection and Labeling Challenges}

The animals in the images can be challenging to detect and classify, even for humans. We have determined that there are six main nuisance factors inherent to camera trap data, which can compound upon each other. Detailed analysis of these challenges can be seen in Fig. 3. When an image is too difficult to classify on its own, biologists will often refer to an easier image in the same sequence and then track motion by flipping between sequence frames in order to generate a label for each frame (e.g. is the animal still present or has it gone off the image plane?). We account for this in our experiments by reporting performance at the frame level and at the sequence level. Considering frame level performance allows us to investigate the limits of current models in exceptionally difficult cases.

\subsection{Annotations}

We collected bounding box annotations on Amazon Mechanical Turk, procuring annotations from at least three and up to ten mturkers for each image for redundancy and accuracy. Workers were asked to draw boxes around all instances of a specific type of animal for each image, determined by what label was given to the sequence by the biologists. We used the crowdsourcing method by Branson et al. [54] to determine ground truth boxes from our collective annotations, and to iteratively collect additional annotations as necessary. We found that bounding box precisions varied based on annotator, and determined that for this data the PascalVOC metric of IoU $\geq 0.5$ is appropriate for the detection experiments (as opposed to the COCO IoU averaging metric).

\subsection{Data Split: Cis- and Trans-}

Our goal is exploring generalization to new (i.e. untrained) locations. Thus, we compare the performance of detection and classification algorithms when they are tested at the same locations where they were trained, vs new locations. For brevity, we refer to locations seen during training as cis-locations and locations not seen during training as trans-locations.

From our pool of 20 locations, we selected 9 locations at random to use as trans-location test data, and a single random location to use as trans-location validation data. From the remaining 10 locations, we use images taken on odd days as cis-location test data. From within the data taken on even days, we randomly select $5 \%$ to be used as cis-location validation data. The remaining data is used for training, with the constraint that training and validation sets do not share the same image sequences. This gives us 13, 553 training images, 3,484 validation and 15,827 test images from cis-locations, and 1,725 val and 23,275 

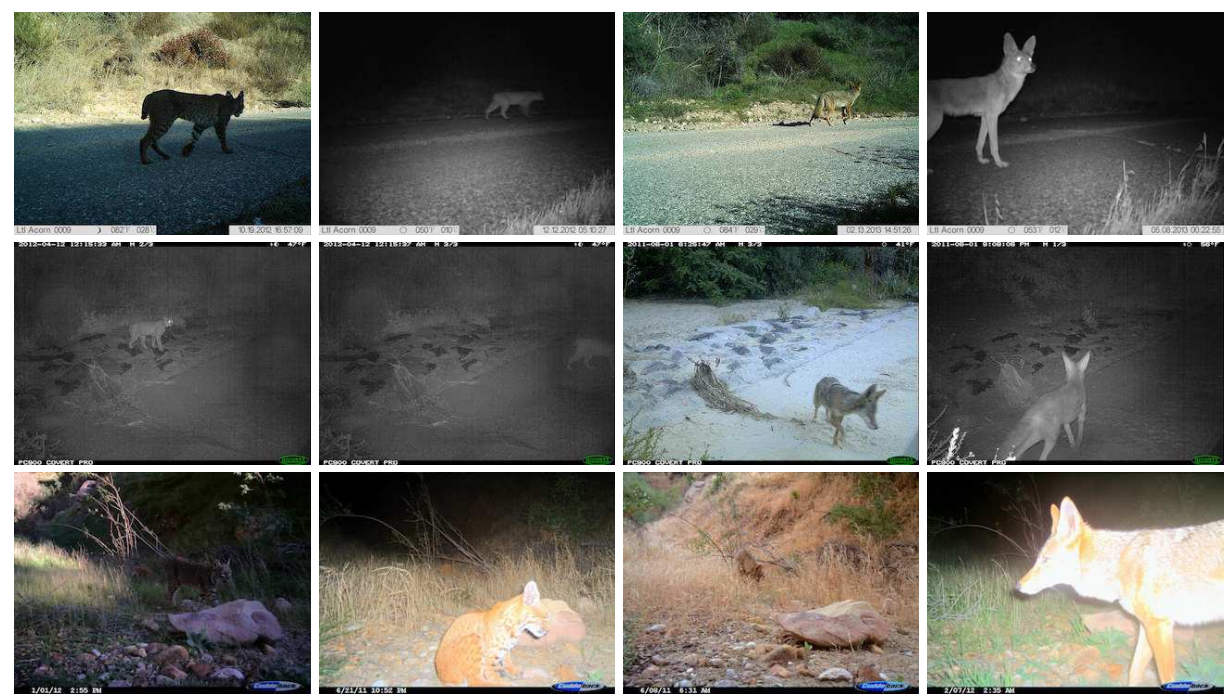

Fig. 2. Camera trap images from three different locations. Each row is a different location and a different camera type. The first two cameras use IR, while the third row used white flash. The first two columns are bobcats, the next two columns are coyotes.

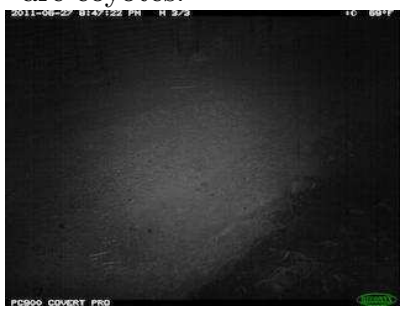

(1) Illumination

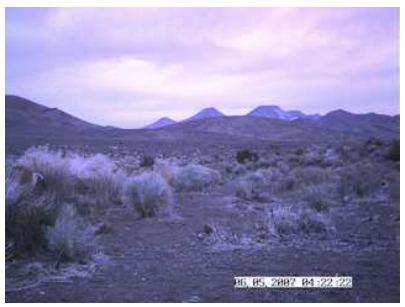

(4) Occlusion

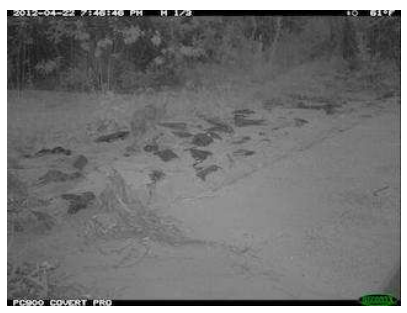

(2) Blur

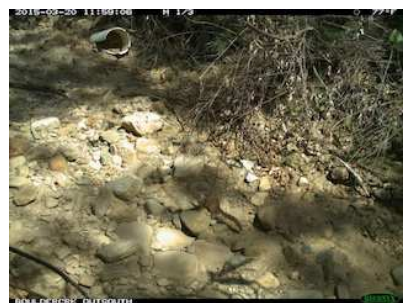

(5) Camouflage

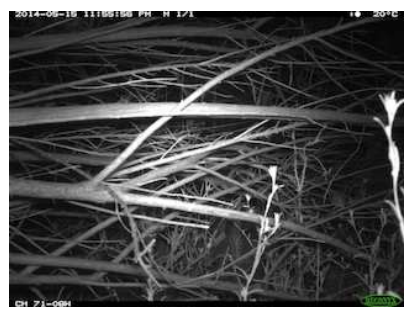

(3) ROI Size

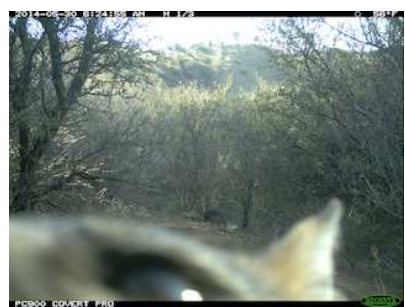

(6) Perspective

Fig. 3. Common data challenges: (1) Illumination: Animals are not always salient. (2) Motion blur: common with poor illumination at night. (3) Size of the region of interest (ROI): Animals can be small or far from the camera. (4) Occlusion: e.g. by bushes or rocks. (5) Camouflage: decreases saliency in animals' natural habitat. (6) Perspective: Animals can be close to the camera, resulting in partial views of the body. 

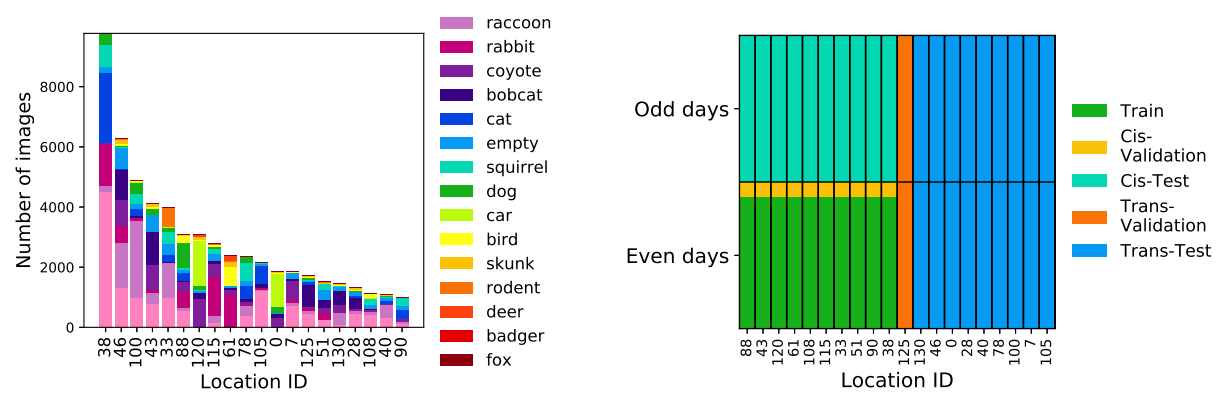

Fig. 4. (Left) Number of annotations for each location, over 16 classes. The ordering of the classes in the legend is from most to least examples overall. The distribution of the species is long-tailed at each location, and each location has a different and peculiar distribution. (Right) Visualization of data splits. "Cis" refers to images from locations seen during training, and "trans" refers to new locations not seen during training.

test images from trans-locations. The data split can be visualized in Fig. 4. We chose to interleave the cis training and test data by day because we found that using a single date to split the data results in additional generalization challenges due to changing vegetation and animal species distributions across seasons. By interleaving, we reduce noise and provide a clean experimental comparison of results on cis- and trans-locations.

\section{Experiments}

Current state-of-the-art computer vision models for classification and detection are designed to work well on test data whose distribution matches the training distribution. However, in our experiments we are explicitly evaluating the models on a different test distribution. In this situation, it is common practice to employ early stopping [55] as a means of preventing overfitting to the train distribution. Therefore, for all classification and detection experiments we monitor performance on both the cis- and trans-location validation sets. In each experiment we save two models, one that we expect has the best performance on the trans-location test set (i.e. a model that generalizes), and one that we expect has the best performance on the cis-location test set (i.e. a model that performs well on the train distribution).

\subsection{Classification}

We explore the generalization of classifiers in 2 different settings: full images and cropped bounding boxes. For each setting we also explore the effects of using and ignoring sequence information. Sequence information is utilized in two different ways: (1) Most Confident we consider the sequence to be classified correctly if the most confident prediction from all frames grouped together is 
correct, or (2) Oracle we consider the sequence to be correctly classified if any frame is correctly classified. Note that (2) is a more optimistic usage of sequence information. For all classification experiments we use an Inception-v3 [56] model pretrained on ImageNet, with an initial learning rate of 0.0045 , rmsprop with a momentum of 0.9 , and a square input resolution of 299 . We employ random cropping (containing at least $65 \%$ of the region), horizontal flipping, and color distortion as data augmentation.

Table 1. Classification top-1 error across experiments. Empty images are removed for these experiments.

\begin{tabular}{|c|cc|cc|cc|}
\cline { 2 - 7 } \multicolumn{1}{c|}{} & \multicolumn{2}{c|}{ Cis-Locations } & \multicolumn{2}{c|}{ Trans-Locations } & \multicolumn{2}{c|}{ Error Increase } \\
\hline Sequence Information & Images & Bboxes & Images & Bboxes & Images & Bboxes \\
\hline None & 19.06 & 8.14 & 41.04 & 19.56 & $115 \%$ & $140 \%$ \\
Most Confident & 17.7 & 7.06 & 34.53 & 15.77 & $95 \%$ & $123 \%$ \\
Oracle & 14.92 & 5.52 & 28.69 & 12.06 & $92 \%$ & $118 \%$ \\
\hline
\end{tabular}

Full Image. We train a classifier on the full images, considering all 15 classes as well as empty images (16 total classes). On the cis-location test set we achieve a top-1 error of $20.83 \%$, and a top- 1 error of $41.08 \%$ on the trans-location test set with a $97 \%$ cis-to-trans increase in error. To investigate if requiring the classifier to both detect and classify animals increased overfitting on the training location backgrounds, we removed the empty images and retrained the classifiers using just the 15 animal classes. Performance stayed at nearly the same levels, with a top-1 error of $19.06 \%$ and $41.04 \%$ for cis- and trans-locations respectively. Utilizing sequence information helped reduce overall error (achieving errors of $14.92 \%$ and $28.69 \%$ on cis- and trans-locations respectively), but even in the most optimistic oracle setting, there is still a $92 \%$ increase in error between evaluating on cis- and trans-locations. See Table 1 for the full results.

Bounding Boxes. We train a classifier on cropped bounding boxes, excluding all empty images (as there is no bounding box in those cases). Using no sequence information we achieve a cis-location top- 1 error of $8.14 \%$ and a trans-location top-1 error of $19.56 \%$. While the overall error has decreased compared to the image level classification, the error increase between cis- and trans-locations is still high at $140 \%$. Sequence information further improved classification results (achieving errors of $5.52 \%$ and $12.06 \%$ on cis- and trans-locations respectively), and slightly reduced generalization error, bringing the error increase down to $118 \%$ in the most optimistic setting. See Table 1 for the full results. Additional experiments investigating the effect of number of images per location, number of training locations, and selection of validation location can be seen in the supplementary material. 
Analysis Fig. 5 provides a high level summary of our experimental findings. Namely, there is a generalization gap between cis- and trans-locations. Cropped boxes help to improve overall performance (shifting the blue lines vertically downward to the red lines), but the gap remains. In the best case scenario (red dashed lines: cropped boxes and optimistically utilizing sequences) we see a $92 \%$ increase in error between the cis- and trans-locations (with the same number of training examples), and 20x increase in training examples to have the same error rate. One might wonder whether this generalization gap is due to a large shift in class distributions between the two locations types. However, Fig. 7 shows that the overall distribution of classes between the locations is similar, and therefore probably does not account for the performance loss.

\subsection{Detection}

We use the Faster-RCNN implementation found in the Tensorflow Object Detection code base [57] as our detection model. We study performance of the FasterRCNN model using two different backbones, ResNet-101 [58] and InceptionResNet-v2 with atrous convolution [57]. Similar to our classification experiments we analyze the effects of using sequence information using two methods: (1) Most Confident we consider a sequence to be labeled correctly if the most confident detection across all frames has an $\mathrm{IoU} \geq 0.5$ with its matched ground truth box; (2) Oracle we consider a sequence to be labeled correctly if any frame's most confident detection has IoU $\geq 0.5$ with its matched ground truth box. Note that method (2) is more optimistic than method (1).

Our detection models are pretrained on COCO [10], images are resized to have a max dimension of 1024 and a minimum dimension of 600; each experiment uses SGD with a momentum of 0.9 and a fixed learning rate schedule. Starting at 0.0003 we decay the learning rate by a factor of 10 at $90 \mathrm{k}$ steps and $120 \mathrm{k}$ steps. We use a batch size of 1 , and employ horizontal flipping for data augmentation. For evaluation, we consider a detected box to be correct if its $\mathrm{IoU} \geq 0.5$ with a ground truth box.

Results from our experiments can be seen in Table 2 and Fig 9. We find that both backbone architectures perform similarly. Without taking sequence information into account, the models achieve $\sim 77 \% \mathrm{mAP}$ on cis-locations and $\sim 71 \%$ $\mathrm{mAP}$ on trans-locations. Adding sequence information using the most confident metric improves results, bringing performance on cis- and trans-locations to similar values at $\sim 85 \%$. Finally, using the oracle metric brings mAP into the $90 \mathrm{~s}$ for both locations. Precision-recall curves at the frame and sequence levels for both detectors can be seen in Fig. 9 .

Analysis There is a significantly lower generalization error in our detection experiments when not using sequences than what we observed in the classification experiments $(\sim 30 \%$ error increase for detections vs $\sim 115 \%$ error increase for classification). When using sequence information, the generalization error for detections is reduced to only $\sim 5 \%$. 

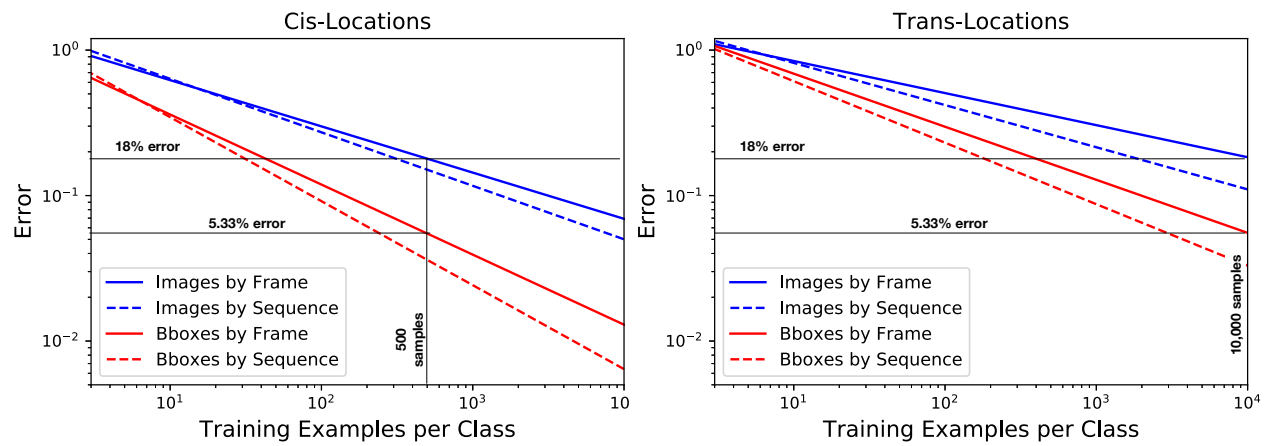

Fig. 5. Classification error vs. number of class-specific training examples. Error is calculated as 1 - AUC (area under the precision-recall curve). Best-fit lines through the error-vs-n.examples points for each class in each scenario (points omitted for clarity), with average $r^{2}=0.261$. An example of line fit on top of data can be seen in Fig. 7. As expected, error decreases as a function of the number of training examples. This is true both for image classification (blue) and bounding-box classification (red) on both cis-locations and trans-locations. However, trans-locations show significantly higher error rates. To operate at an error rate of $5.33 \%$ on bounding boxes or $18 \%$ on images at the cis-locations we need 500 training examples, while we need 10,000 training examples to achieve the same error rate at the trans-locations, a 20x increase in data.
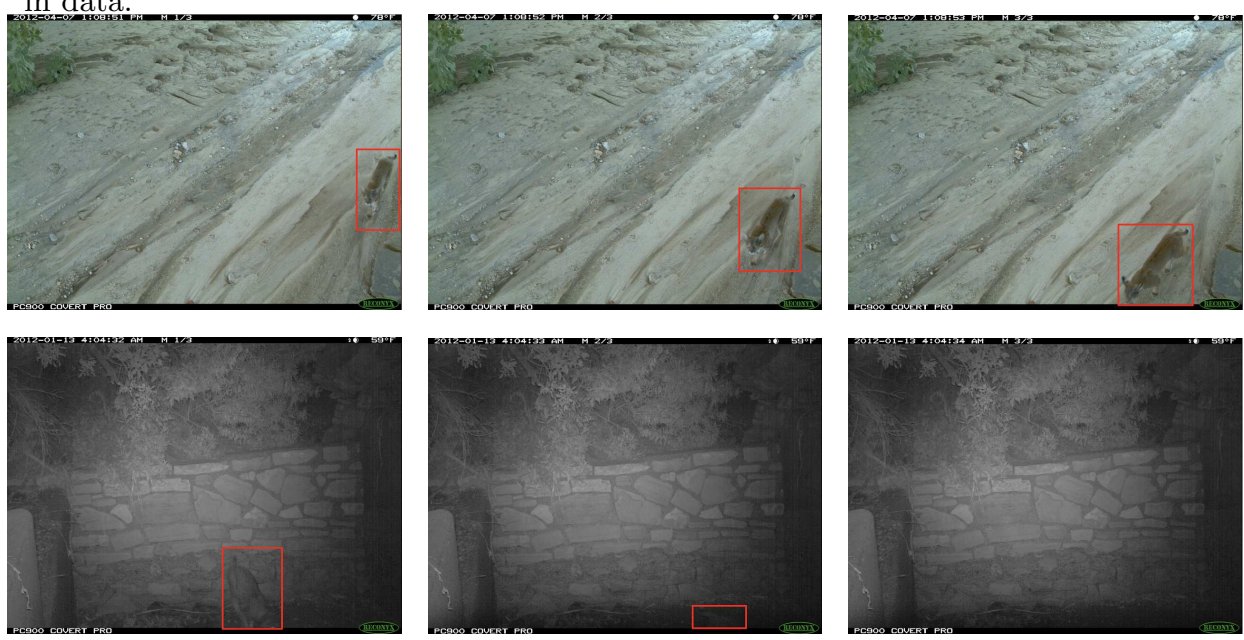

Fig. 6. Trans-classification failure cases at the sequence level: (Based on classification of bounding box crops) In the first sequence, the network struggles to distinguish between 'cat' and 'bobcat', incorrectly predicting 'cat' in all three images with a mean confidence of 0.82 . In the second sequence, the network struggles to classify a bobcat at an unfamiliar pose in the first image and instead predicts 'raccoon' with a confidence of 0.84 . Little additional sequence information is available in this case, as the next frame contains only a blurry tail, and the last frame is empty 

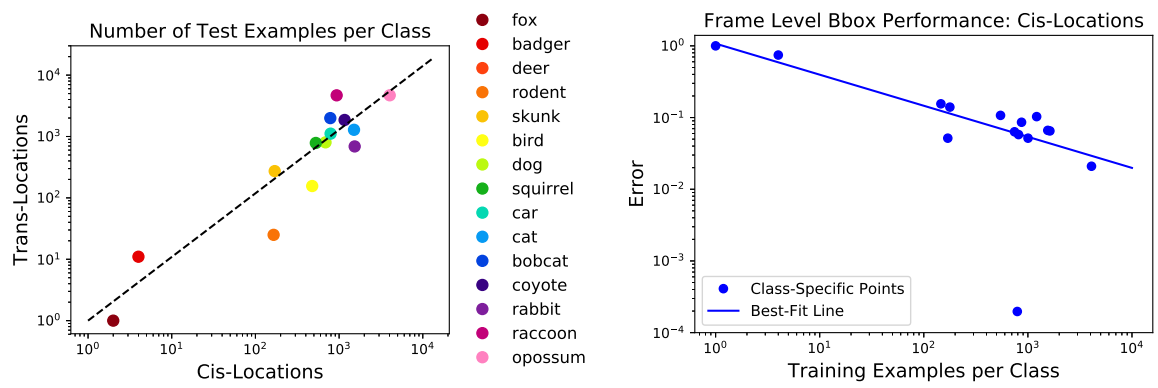

Fig. 7. (Left) Distribution of species across the two test sets. (Right) An example of line fit used to generate the plots in Fig. 5

Qualitatively, we found the mistakes can often be attributed to nuisance factors that make frames difficult. We see examples of all 6 nuisance factors described in Fig. 3 causing detection failures. The errors remaining at the sequence level occur when these nuisance factors are present in all frames of a sequence, or when the sequence only contains a single, challenging frame containing an animal. Examples of sequence-level detection failures can be seen in Fig. 8. The generalization gap at the frame level implies that our models are better able to deal with nuisance factors at locations seen during training.

Our experiments show that there is a small generalization gap when we use sequence information. However, overall performance has not saturated, and current state-of-the-art detectors are not achieving high precision at high recall values $(1 \%$ precision at recall $=95 \%)$. So while we are encouraged by the results, there is still room for improvement. When we consider frames independently, we see that the generalization gap reappears. Admittedly this is a difficult case as it is not clear what the performance of a human would be without sequence information. However, we know that there are objects that can be detected in these frames and this dataset will challenge the next generation of detection models to accurately localize these difficult cases.

Table 2. Detection mAP at $\mathrm{IoU}=0.5$ across experiments.

\begin{tabular}{|c|cc|cc|cc|}
\cline { 2 - 7 } \multicolumn{1}{c|}{} & \multicolumn{2}{c|}{ Cis-Locations } & \multicolumn{2}{c|}{ Trans-Locations } & \multicolumn{2}{c|}{ Error Increase } \\
\hline Sequence Information & ResNet & Inception & ResNet & Inception & ResNet & Inception \\
\hline None & 77.10 & 77.57 & 70.17 & 71.37 & $30 \%$ & $27.6 \%$ \\
Most Confident & 84.78 & 86.22 & 84.09 & 85.44 & $4.5 \%$ & $5.6 \%$ \\
Oracle & 94.95 & 95.04 & 92.13 & 93.09 & $55.8 \%$ & $39.3 \%$ \\
\hline
\end{tabular}



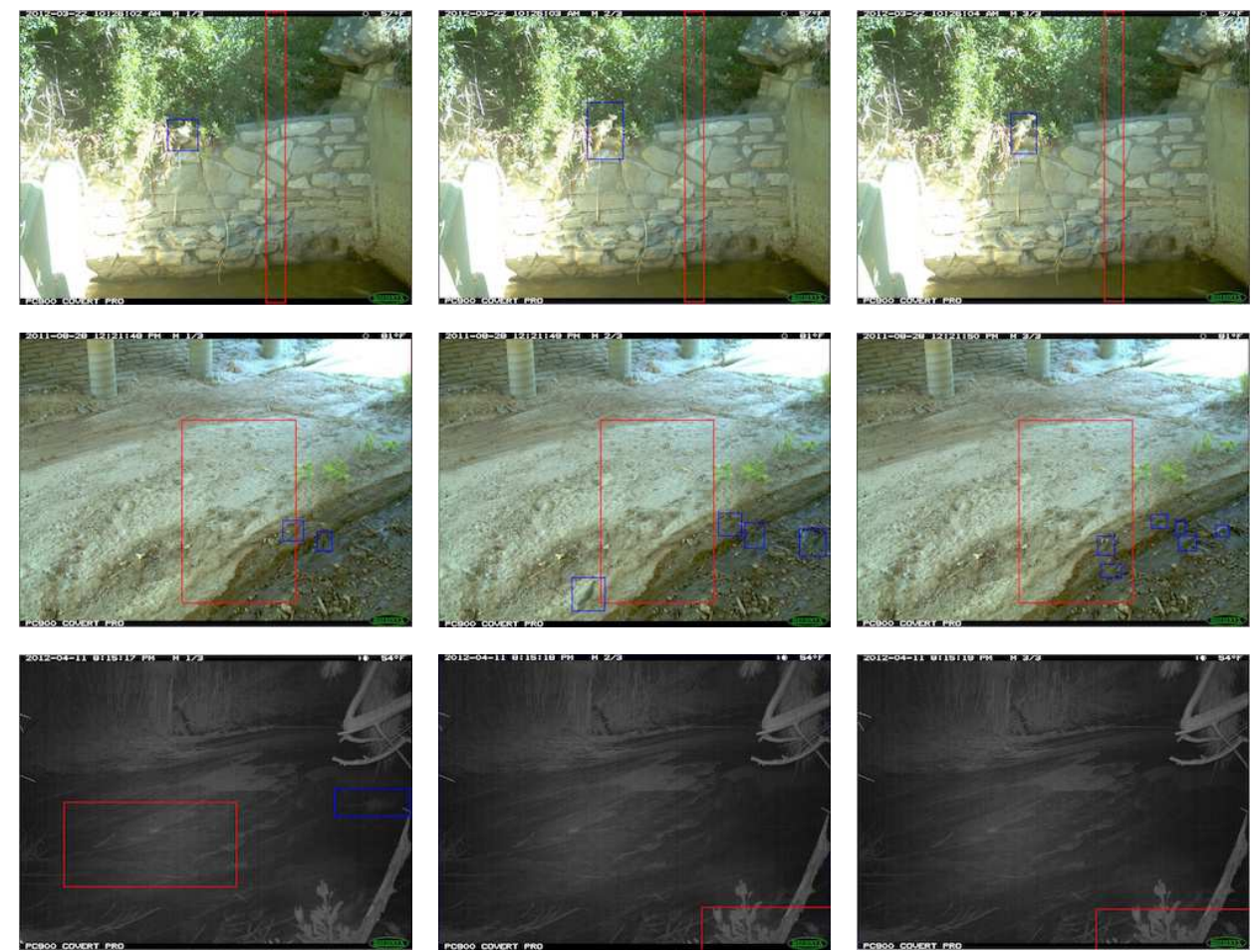

Fig. 8. Trans-detection failure cases at the sequence level: Highest-confidence detection in red, ground truth in blue. In all cases the confidence of the detection was lower than 0.2. The first two sequences have small ROI, compounded with challenging lighting in the first and camouflaged birds in the second. In the third the opossum is poorly illuminated and only visible in the first frame.
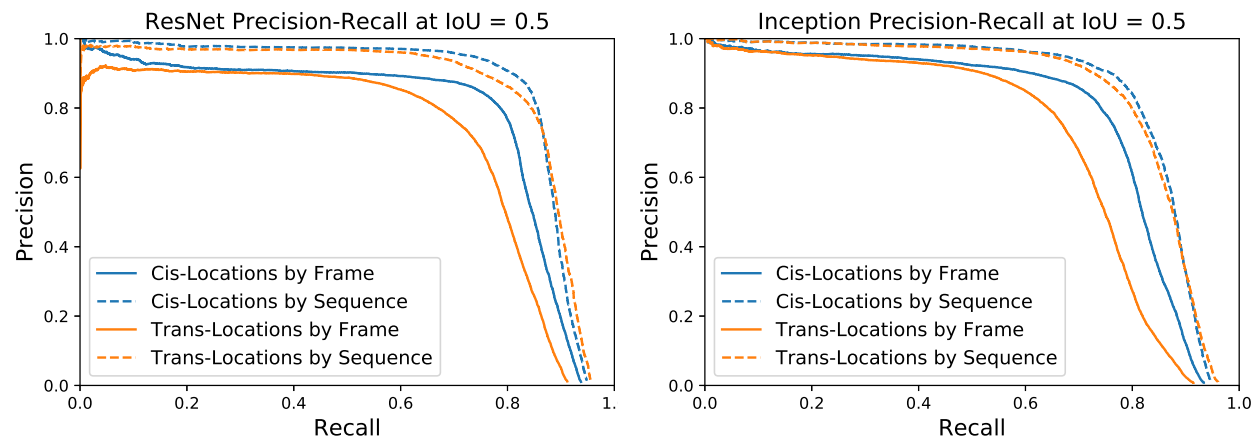

Fig. 9. Faster-RCNN precision-recall curves at an IoU of 0.5 , by frame and by sequence, using a confidence-based approach to determine which frame should represent the sequence 


\section{Conclusions}

The question of generalization to novel image statistics is taking center stage in visual recognition. Many indicators point to the fact that current systems are data-inefficient and do not generalize well to new scenarios. Current systems are, in essence, glorified pattern-matching machines, rather than intelligent visual learners.

Many problem domains face a generalization challenge where the test conditions are potentially highly different than what has been seen during training. Self driving cars navigating new cities, rovers exploring new planets, security cameras installed in new buildings, and assistive technologies installed in new homes are all examples where good generalization is critical for a system to be useful. However, the most popular detection and classification benchmark datasets $[9,10,28,29]$ are evaluating models on test distributions that are the same as the train distributions. Clearly it is important for models to do well on data coming from the same distribution as the train set. However, we argue that it is important to characterize the generalization behavior of these models when the test distribution deviates from the train distribution. Current datasets do not allow researchers to quantify the generalization behavior of their models.

We contribute a new dataset and evaluation protocol designed specifically to analyze the generalization behavior of classification and detection models. Our experiments reveal that there is room for significant improvement on the generalization of state-of-the-art classification models. Detection helps to improve overall classification accuracy, and we find that while detectors generalize better to new locations, there is room to improve their precision at high recall rates.

Camera traps provide a unique experimental setup that allow us to explore the generalization of models while controlling for many nuisance factors. Our current dataset is already revealing interesting behaviors of classification and detection models. There is still more information that we can learn by expanding our dataset in both data quantity and evaluation metrics. We plan to extend this dataset by adding additional locations, both from the American Southwest and from new regions. Drastic landscape and vegetation changes will allow us to investigate generalization in an even more challenging setting. Rare and novel events are frequently the most important and most challenging to detect and classify, and while our dataset already has these properties, we plan to define experimental protocols and data splits for benchmarking low-shot performance and the open-set problem of detecting and/or classifying species not seen during training.

\section{Acknowledgements}

We would like to thank the USGS and NPS for providing data. This work was supported by NSFGRFP Grant No. 1745301, the views are those of the authors and do not necessarily reflect the views of the NSF. Compute time was provided by an AWS Research Grant. 


\section{References}

1. Van Horn, G., Mac Aodha, O., Song, Y., Shepard, A., Adam, H., Perona, P., Belongie, S.: The inaturalist challenge 2017 dataset. arXiv preprint arXiv:1707.06642 (2017)

2. Norouzzadeh, M.S., Nguyen, A., Kosmala, M., Swanson, A., Packer, C., Clune, J.: Automatically identifying wild animals in camera trap images with deep learning. arXiv preprint arXiv:1703.05830 (2017)

3. van Horn, G., Barry, J., Belongie, S., Perona, P.: The Merlin Bird ID smartphone app (http://merlin.allaboutbirds.org/download/).

4. Esteva, A., Kuprel, B., Novoa, R.A., Ko, J., Swetter, S.M., Blau, H.M., Thrun, S.: Dermatologist-level classification of skin cancer with deep neural networks. Nature 542(7639) (2017) 115

5. Poplin, R., Varadarajan, A.V., Blumer, K., Liu, Y., McConnell, M.V., Corrado, G.S., Peng, L., Webster, D.R.: Prediction of cardiovascular risk factors from retinal fundus photographs via deep learning. Nature Biomedical Engineering (2018) 1

6. Fukushima, K., Miyake, S.: Neocognitron: A self-organizing neural network model for a mechanism of visual pattern recognition. In: Competition and cooperation in neural nets. Springer (1982) 267-285

7. LeCun, Y., Bottou, L., Bengio, Y., Haffner, P.: Gradient-based learning applied to document recognition. Proceedings of the IEEE 86(11) (1998) 2278-2324

8. Schaller, R.R.: Moore's law: past, present and future. IEEE spectrum 34(6) (1997) 52-59

9. Deng, J., Dong, W., Socher, R., Li, L.J., Li, K., Fei-Fei, L.: ImageNet: A LargeScale Hierarchical Image Database. In: CVPR09. (2009)

10. Lin, T.Y., Maire, M., Belongie, S., Hays, J., Perona, P., Ramanan, D., Dollár, P., Zitnick, C.L.: Microsoft coco: Common objects in context. In: European conference on computer vision, Springer (2014) 740-755

11. Van Horn, G., Perona, P.: The devil is in the tails: Fine-grained classification in the wild. arXiv preprint arXiv:1709.01450 (2017)

12. Pan, S.J., Yang, Q.: A survey on transfer learning. IEEE Transactions on knowledge and data engineering 22(10) (2010) 1345-1359

13. Torralba, A., Efros, A.A.: Unbiased look at dataset bias. In: Computer Vision and Pattern Recognition (CVPR), 2011 IEEE Conference on, IEEE (2011) 1521-1528

14. Welinder, P., Welling, M., Perona, P.: A lazy man's approach to benchmarking: Semisupervised classifier evaluation and recalibration. In: Computer Vision and Pattern Recognition (CVPR), 2013 IEEE Conference on, IEEE (2013) 3262-3269

15. Murphy, G.: The big book of concepts. MIT press (2004)

16. Ren, X., Han, T.X., He, Z.: Ensemble video object cut in highly dynamic scenes. In: Computer Vision and Pattern Recognition (CVPR), 2013 IEEE Conference on, IEEE (2013) 1947-1954

17. Yu, X., Wang, J., Kays, R., Jansen, P.A., Wang, T., Huang, T.: Automated identification of animal species in camera trap images. EURASIP Journal on Image and Video Processing 2013(1) (2013) 52

18. Wilber, M.J., Scheirer, W.J., Leitner, P., Heflin, B., Zott, J., Reinke, D., Delaney, D.K., Boult, T.E.: Animal recognition in the mojave desert: Vision tools for field biologists. In: Applications of Computer Vision (WACV), 2013 IEEE Workshop on, IEEE (2013) 206-213

19. Chen, G., Han, T.X., He, Z., Kays, R., Forrester, T.: Deep convolutional neural network based species recognition for wild animal monitoring. In: Image Processing (ICIP), 2014 IEEE International Conference on, IEEE (2014) 858-862 
20. Lin, K.H., Khorrami, P., Wang, J., Hasegawa-Johnson, M., Huang, T.S.: Foreground object detection in highly dynamic scenes using saliency. In: Image Processing (ICIP), 2014 IEEE International Conference on, IEEE (2014) 1125-1129

21. Swanson, A., Kosmala, M., Lintott, C., Simpson, R., Smith, A., Packer, C.: Snapshot serengeti, high-frequency annotated camera trap images of 40 mammalian species in an african savanna. Scientific data 2 (2015) 150026

22. Zhang, Z., Han, T.X., He, Z.: Coupled ensemble graph cuts and object verification for animal segmentation from highly cluttered videos. In: Image Processing (ICIP), 2015 IEEE International Conference on, IEEE (2015) 2830-2834

23. Zhang, Z., He, Z., Cao, G., Cao, W.: Animal detection from highly cluttered natural scenes using spatiotemporal object region proposals and patch verification. IEEE Transactions on Multimedia 18(10) (2016) 2079-2092

24. Miguel, A., Beery, S., Flores, E., Klemesrud, L., Bayrakcismith, R.: Finding areas of motion in camera trap images. In: Image Processing (ICIP), 2016 IEEE International Conference on, IEEE (2016) 1334-1338

25. Giraldo-Zuluaga, J.H., Salazar, A., Gomez, A., Diaz-Pulido, A.: Camera-trap images segmentation using multi-layer robust principal component analysis. The Visual Computer (2017) 1-13

26. Yousif, H., Yuan, J., Kays, R., He, Z.: Fast human-animal detection from highly cluttered camera-trap images using joint background modeling and deep learning classification. In: Circuits and Systems (ISCAS), 2017 IEEE International Symposium on, IEEE (2017) 1-4

27. Villa, A.G., Salazar, A., Vargas, F.: Towards automatic wild animal monitoring: Identification of animal species in camera-trap images using very deep convolutional neural networks. Ecological Informatics 41 (2017) 24-32

28. Everingham, M., Van Gool, L., Williams, C.K., Winn, J., Zisserman, A.: The pascal visual object classes (voc) challenge. International journal of computer vision 88(2) (2010) 303-338

29. Krasin, I., Duerig, T., Alldrin, N., Ferrari, V., Abu-El-Haija, S., Kuznetsova, A., Rom, H., Uijlings, J., Popov, S., Veit, A., Belongie, S., Gomes, V., Gupta, A., Sun, C., Chechik, G., Cai, D., Feng, Z., Narayanan, D., Murphy, K.: Openimages: A public dataset for large-scale multi-label and multi-class image classification. Dataset available from https://github.com/openimages (2017)

30. Ponce, J., Berg, T.L., Everingham, M., Forsyth, D.A., Hebert, M., Lazebnik, S., Marszalek, M., Schmid, C., Russell, B.C., Torralba, A., et al.: Dataset issues in object recognition. In: Toward category-level object recognition. Springer (2006) 29-48

31. Spain, M., Perona, P.: Some objects are more equal than others: Measuring and predicting importance. In: European Conference on Computer Vision (ECCV), Springer (2008) 523-536

32. Wah, C., Branson, S., Welinder, P., Perona, P., Belongie, S.: The caltech-ucsd birds-200-2011 dataset. (2011)

33. Nilsback, M.E., Zisserman, A.: A visual vocabulary for flower classification. In: Proceedings of the IEEE Conference on Computer Vision and Pattern Recognition. Volume 2. (2006) 1447-1454

34. Kumar, N., Belhumeur, P.N., Biswas, A., Jacobs, D.W., Kress, W.J., Lopez, I., Soares, J.V.B.: Leafsnap: A computer vision system for automatic plant species identification. In: The 12th European Conference on Computer Vision (ECCV). (October 2012) 
35. Van Horn, G., Branson, S., Farrell, R., Haber, S., Barry, J., Ipeirotis, P., Perona, P., Belongie, S.: Building a bird recognition app and large scale dataset with citizen scientists: The fine print in fine-grained dataset collection. In: Proceedings of the IEEE Conference on Computer Vision and Pattern Recognition. (2015) 595-604

36. St-Charles, P.L., Bilodeau, G.A., Bergevin, R.: Subsense: A universal change detection method with local adaptive sensitivity. IEEE Transactions on Image Processing 24(1) (2015) 359-373

37. Babaee, M., Dinh, D.T., Rigoll, G.: A deep convolutional neural network for background subtraction. arXiv preprint arXiv:1702.01731 (2017)

38. Zhan, Y., Fu, K., Yan, M., Sun, X., Wang, H., Qiu, X.: Change detection based on deep siamese convolutional network for optical aerial images. IEEE Geoscience and Remote Sensing Letters 14(10) (2017) 1845-1849

39. Benedek, C., Szirányi, T.: A mixed markov model for change detection in aerial photos with large time differences. In: Pattern Recognition, 2008. ICPR 2008. 19th International Conference on, IEEE (2008) 1-4

40. : emammal: a tool for collecting, archiving, and sharing camera trapping images and data. https://emammal.si.edu/ Accessed: 2018-03-13.

41. Csurka, G.: Domain adaptation for visual applications: A comprehensive survey. arXiv preprint arXiv:1702.05374 (2017)

42. Ganin, Y., Lempitsky, V.: Unsupervised domain adaptation by backpropagation. In: International Conference on Machine Learning. (2015) 1180-1189

43. Gebru, T., Hoffman, J., Fei-Fei, L.: Fine-grained recognition in the wild: A multitask domain adaptation approach. In: 2017 IEEE International Conference on Computer Vision (ICCV), IEEE (2017) 1358-1367

44. Busto, P.P., Gall, J.: Open set domain adaptation. In: The IEEE International Conference on Computer Vision (ICCV). Volume 1. (2017)

45. Hoffman, J., Wang, D., Yu, F., Darrell, T.: Fcns in the wild: Pixel-level adversarial and constraint-based adaptation. arXiv preprint arXiv:1612.02649 (2016)

46. Chen, Y., Li, W., Van Gool, L.: Road: Reality oriented adaptation for semantic segmentation of urban scenes. arXiv preprint arXiv:1711.11556 (2017)

47. Zhang, Y., David, P., Gong, B.: Curriculum domain adaptation for semantic segmentation of urban scenes. In: The IEEE International Conference on Computer Vision (ICCV). Volume 2. (2017) 6

48. Peng, X., Sun, B., Ali, K., Saenko, K.: Learning deep object detectors from 3d models. In: Computer Vision (ICCV), 2015 IEEE International Conference on, IEEE (2015) 1278-1286

49. Tang, K., Ramanathan, V., Fei-Fei, L., Koller, D.: Shifting weights: Adapting object detectors from image to video. In: Advances in Neural Information Processing Systems. (2012) 638-646

50. Sun, B., Saenko, K.: From virtual to reality: Fast adaptation of virtual object detectors to real domains. In: BMVC. Volume 1. (2014) 3

51. Hattori, H., Boddeti, V.N., Kitani, K., Kanade, T.: Learning scene-specific pedestrian detectors without real data. In: Computer Vision and Pattern Recognition (CVPR), 2015 IEEE Conference on, IEEE (2015) 3819-3827

52. Xu, J., Ramos, S., Vázquez, D., López, A.M.: Domain adaptation of deformable part-based models. IEEE transactions on pattern analysis and machine intelligence 36(12) (2014) 2367-2380

53. Raj, A., Namboodiri, V.P., Tuytelaars, T.: Subspace alignment based domain adaptation for rcnn detector. arXiv preprint arXiv:1507.05578 (2015)

54. Grant Van Horn, Scott Laurie, S.B., Perona, P.: Lean multiclass crowdsourcing. Computer Vision and Pattern Recognition (2018) 
55. Bengio, Y.: Practical recommendations for gradient-based training of deep architectures. In: Neural networks: Tricks of the trade. Springer (2012) 437-478

56. Szegedy, C., Vanhoucke, V., Ioffe, S., Shlens, J., Wojna, Z.: Rethinking the inception architecture for computer vision. In: Proceedings of the IEEE Conference on Computer Vision and Pattern Recognition. (2016) 2818-2826

57. Huang, J., Rathod, V., Sun, C., Zhu, M., Korattikara, A., Fathi, A., Fischer, I., Wojna, Z., Song, Y., Guadarrama, S., et al.: Speed/accuracy trade-offs for modern convolutional object detectors. In: IEEE CVPR. (2017)

58. He, K., Zhang, X., Ren, S., Sun, J.: Deep residual learning for image recognition. In: Proceedings of the IEEE conference on computer vision and pattern recognition. (2016) 770-778 